PROCEEDINGS OF THE

AMERICAN MATHEMATICAL SOCIETY

Volume 127, Number 3, March 1999, Pages 745-751

S 0002-9939(99)04581-5

\title{
FACTORIZATION OF FUNCTIONS IN GENERALIZED NEVANLINNA CLASSES
}

\author{
CHARLES HOROWITZ
}

(Communicated by Theodore W. Gamelin)

\begin{abstract}
For functions in the classical Nevanlinna class analytic projection of $\log \left|f\left(e^{i \theta}\right)\right|$ produces $\log F(z)$ where $F$ is the outer part of $f$; i.e., this projection factors out the inner part of $f$. We show that if $\log |f(z)|$ is area integrable with respect to certain measures on the disc, then the appropriate analytic projections of $\log |f|$ factor out zeros by dividing $f$ by a natural product which is a disc analogue of the classical Weierstrass product. This result is actually a corollary of a more general theorem of M. Andersson. Our contribution is to give a simple one complex variable proof which accentuates the connection with the Weierstrass product and other canonical objects of complex analysis.
\end{abstract}

A function $f$ analytic in the unit disc $U$ is said to belong to the Nevanlinna class $N$ if

$$
\sup _{0<r<1} \int_{0}^{2 \pi} \log ^{+}\left|f\left(r e^{i \theta}\right)\right| d \theta<\infty .
$$

By a classical result, every $f \in N$ has a canonical factorization $f=I O$ where $I$ is an inner function and $O$ is an outer function. As is well known $\log |O(z)|$ is just the Poisson integral of the boundary function $\log \left|f\left(e^{i \theta}\right)\right|$. In other words $\log O(z)$ is the analytic projection of $\log \left|f\left(e^{i \theta}\right)\right|$. Qualitatively, the analytic projection of $\log \left|f\left(e^{i \theta}\right)\right|$ "divides out" the inner part of $f$.

We wish to consider an analogous process in the generalized Nevanlinna classes $\mathbb{A}_{p, \alpha}(U)(1 \leq p<\infty, 0 \leq \alpha<\infty)$ of functions analytic in $U$ such that

$$
\|f\|_{\mathbb{A}_{p, \alpha}}^{p}=\frac{1}{\pi} \int_{U}|\log | f(z)||^{p}\left(1-|z|^{2}\right)^{\alpha} d A(z)<\infty .
$$

Here $d A(z)$ is just the regular Lebesgue measure on $U$. For $p=1$, it is easily verified that the defining condition is equivalent to the condition

$$
\int \log ^{+}|f(z)|\left(1-|z|^{2}\right)^{\alpha} d A(z)<\infty .
$$

The classes $\mathbb{A}_{p, \alpha}$ have been considered by several authors (see [4], [5], [6], [8]). In particular, in [6], the authors give a complete description of zero sets for the classes

Received by the editors June 12, 1997.

1991 Mathematics Subject Classification. Primary 30D50.

(C)1999 American Mathematical Society 
$\mathbb{A}_{p, 0}$. For our purposes it will suffice to use the following weaker necessary condition:

(0) For $f$ analytic in $U$ let $\left\{z_{k}\right\}$ denote the zero set of $f$; each zero is repeated according to its multiplicity. Then if $f \in \mathbb{A}_{p, \alpha}(0 \leq \alpha, 1 \leq p<\infty)$, $\sum_{k}\left(1-\left|z_{k}\right|\right)^{2+\alpha}<\infty$.

In [8] and [9] this was proved for $p=1$ and all $\alpha$. For general $p>1$ it follows from the inclusion $\mathbb{A}_{p, \alpha} \subset \mathbb{A}_{1, \alpha}$.

In [10] it was proved that for every $\alpha>0$

$$
\hat{K}_{\alpha}(z, \zeta)=\frac{\alpha+1}{\pi} \frac{\left(1-|\zeta|^{2}\right)^{\alpha}}{(1-\bar{\zeta} z)^{\alpha+2}}
$$

is a reproducing kernel for analytic functions in $U$. It follows easily that the kernel

$$
\operatorname{Re} \frac{\alpha+1}{\pi}\left(\frac{2}{(1-\bar{\zeta} z)^{\alpha+2}}-1\right)
$$

provides an orthogonal projector from the space $L^{2}\left(\left(1-|\zeta|^{2}\right)^{\alpha}\right) d A(\zeta)$ onto the subspace of harmonic elements, and that the kernel

$$
K_{\alpha}(z, \zeta)=\frac{(\alpha+1)}{\pi}\left(\frac{2}{(1-\bar{\zeta} z)^{\alpha+2}}-1\right)
$$

projects real functions in $L^{2}\left(\left(1-|\zeta|^{2}\right)^{\alpha} d A(\zeta)\right)$ onto the analytic completion of their harmonic projection. See also [3] and [7] for generalizations of these facts.

In this note we shall show that if $f$ belongs to $\mathbb{A}_{p, n}$ for some $p \geq 1$ and integer $n \geq 0$, then the projection of $\log |f|$ using the kernel $K_{n}$ in (2) divides $f$ by a natural product which is a disc analogue of the classical Weierstrass product. This result can be obtained as a corollary of a more general theorem of M. Andersson ([1] and [2]). Our contribution is to give a simple one complex variable proof which accentuates the connection with Weierstrass products, Blaschke products, and other canonical objects of complex analysis.

This note was originally written without knowledge of Andersson's work. The author is indebted to the referee and also to Professor J. Bruna for drawing his attention to Andersson's important results.

Our main result will be developed through a series of lemmas. The first is well known and follows directly from Green's theorem.

Lemma 1. Let $g \in C^{2}(\bar{U})$ such that $g(z)=O\left((1-|z|)^{2}\right)$ as $|z| \rightarrow 1$. Let $f$ be analytic in a neighborhood of $\bar{U}$ and nonvanishing on $|z|=1$. Then if $z_{1} \ldots z_{n}$ are the zeros of $f$ in $U$, repeated according to multiplicity,

$$
\frac{1}{2 \pi} \int_{U} \Delta g(z) \log |f(z)| d A(z)=\sum_{k=1}^{n} g\left(z_{k}\right) .
$$

In terms of distributions Lemma 1 is essentially equivalent to the statement $\Delta \log \left|z-z_{0}\right|=2 \pi \delta_{z_{0}}$. We shall need the following generalization of Lemma 1.

Lemma 2. Let $f$ be analytic in $U$ such that for some $m \geq 0\left(1-|z|^{2}\right)^{m} \log |f(z)|$ is integrable, i.e., $f \in \mathbb{A}_{1, m}$, and let $\left\{z_{k}\right\}$ designate the zeros of $f$, repeated according to multiplicity. Now assume that $g \in C^{2}(\bar{U})$ satisfies

$$
g(z)=O\left(\left(1-|z|^{2}\right)^{m+2}\right) .
$$


Then

$$
\frac{1}{2 \pi} \int_{U} \Delta g(z) \log |f(z)| d A(z)=\sum_{k} g\left(z_{k}\right) .
$$

Proof. Choose a sequence $r_{\ell} \nearrow 1$ such that on the circles $|z|=r_{\ell}, f$ is nonvanishing. Thus by Lemma 1 we have for each $\ell$

$$
\frac{1}{2 \pi} \int_{U} \Delta g(z) \log \left|f\left(r_{\ell} z\right)\right| d A(z)=\sum_{\left|z_{k}\right| \leq r_{\ell}} g\left(\frac{z_{k}}{r_{\ell}}\right) .
$$

Now by our hypotheses $\log |f(z)|$ is integrable with respect to the measure

$$
|\Delta g(z)| d A(z) .
$$

Since translation is continuous in the $L^{1}$ norm the left side of (4) tends to the left side of (3) as $\ell \rightarrow \infty$.

Turning to the right side of (4), in view of (0), our hypotheses ensure that $\sum_{k}\left(1-\left|z_{k}\right|\right)^{m+2}<\infty$, which implies that $\sum_{k}\left|g\left(z_{k}\right)\right|<\infty$. Now it follows easily from the continuity of $g$ that

$$
\lim _{\ell \rightarrow \infty} \sum_{\left|z_{k}\right|<r_{\ell}} g\left(\frac{z_{k}}{r_{\ell}}\right)=\sum_{k} g\left(z_{k}\right),
$$

completing the proof of the lemma.

Definition 1. For $z, w \in U$ define $B_{z}(w)=\bar{z} \frac{(z-w)}{1-\bar{z} w}$.

Definition 2. For $z, w \in U$ and an integer $n \geq 0$ let

$$
\begin{aligned}
& h_{n}(z, w)=\sum_{k=1}^{n+1} \frac{\left(1-B_{z}(w)\right)^{k}}{k}, \\
& g_{n}(z, w)=B_{z}(w) \exp h_{n}(z, w) .
\end{aligned}
$$

Lemma 3. For every $z, w$ and $n$ the following properties hold:

(a) $B_{z}(w) \frac{\partial^{2}}{\partial z \partial \bar{z}} B_{z}(w)=\frac{\partial}{\partial z} B_{z}(w) \frac{\partial}{\partial \bar{z}} B_{z}(w)$.

(b) $\frac{\partial^{2}}{\partial \bar{z} \partial z} h_{n}(z, w)=-(n+1)\left(\frac{1-|z|^{2}}{1-\bar{z} w}\right)^{n} \frac{1}{(1-\bar{z} w)^{2}}$.

(c) For each fixed $w$ and $n, \log \left|g_{n}(z, w)\right|=O\left((1-|z|)^{n+2}\right)$ as $|z| \rightarrow 1$.

Proof. (a) By calculation,

$$
\frac{\partial}{\partial z} B_{z}(w)=\frac{\bar{z}}{1-\bar{z} w} ; \quad \frac{\partial}{\partial \bar{z}} B_{z}(w)=\frac{z-w}{(1-\bar{z} w)^{2}}
$$

and

$$
\frac{\partial}{\partial \bar{z}} \frac{\partial}{\partial z} B_{z}(w)=\frac{1}{(1-\bar{z} w)^{2}},
$$

from which (a) follows immediately. 
(b) From the definition of $h_{n}(z, w)$, if $B_{z}(w) \neq 0$, then

$$
\begin{aligned}
\frac{\partial}{\partial z} h_{n} & =\left[\sum_{k=1}^{n+1}\left[1-B_{z}(w)\right]^{k-1}\right]\left(-\frac{\partial}{\partial z} B_{z}(w)\right) \\
& =(\text { by summing a geometric series }) \\
& \frac{1-\left[1-B_{z}(w)\right]^{n+1}}{B_{z}(w)}\left(-\frac{\partial}{\partial z} B_{z}(w)\right) .
\end{aligned}
$$

So

$$
\begin{aligned}
& \frac{\partial^{2}}{\partial \bar{z} \partial z} h_{n}(z, w)=\frac{1-\left[1-B_{z}(w)\right]^{n+1}}{B_{z}(w)}\left(-\frac{\partial^{2}}{\partial \bar{z} \partial z} B_{z}(w)\right) \\
& \quad+\frac{\left.(n+1) B_{z}(w)\left[1-B_{z}(w)\right]^{n+1}-1+\left[1-B_{z}(w)\right)\right]^{n+1}}{B_{z}^{2}(w)}\left(-\frac{\partial B_{z}(w)}{\partial z} \frac{\partial B_{z}(w)}{\partial \bar{z}}\right) .
\end{aligned}
$$

By (a) this reduces to

$$
\begin{aligned}
\frac{\partial^{2}}{\partial \bar{z} \partial z} h_{n}(z, w) & =-(n+1)\left(1-B_{z}(w)\right)^{n} \frac{\partial^{2}}{\partial \bar{z} \partial z} B_{z}(w) \\
& =-(n+1)\left(\frac{1-|z|^{2}}{1-\bar{z} w}\right)^{n} \frac{1}{(1-\bar{z} w)^{2}} .
\end{aligned}
$$

When $B_{z}(w)=0$ the same result follows by continuity.

(c) Since $h_{n}(z, w)$ is just a Taylor expansion of $-\log B_{z}(w)$ for $B_{z}(w)$ near 1 and since $1-B_{z}(w)=\frac{1-|z|^{2}}{1-\bar{z} w}$, Taylor's theorem with remainder implies (c).

Proposition 1. Let $f$ be an analytic function in $U$ such that for some integer $n \geq 0$ and some $p>0 f \in \mathbb{A}_{p, n}$, and let $\left\{z_{k}\right\}$ be the zeros of $f$ in $U$, repeated according to their multiplicities. Assume $f(0) \neq 0$, and define

$$
P(w)=\prod_{k=1}^{\infty} g_{n}\left(z_{k}, w\right) \quad\left(g_{n} \text { as in Definition } 2\right) .
$$

Then $P(w)$ is a well-defined analytic function in $U$ as is $f(w) / P(w)$ and we have

(6)

$$
\begin{aligned}
\log \mid & f(w) \frac{\sqrt{P(o)}}{P(w)} \mid \\
& =\frac{n+1}{\pi} \int_{U}\left[\frac{1}{(1-\bar{z} w)^{n+2}}+\frac{1}{(1-z \bar{w})^{n+2}}-1\right] \log |f(z)|\left(1-|z|^{2}\right)^{n} d A(z) .
\end{aligned}
$$

Proof. Since $f \in \mathbb{A}_{p, n}$ we have by (0) that

$$
\sum\left(1-\left|z_{k}\right|\right)^{n+2}<\infty
$$

Using the result of Lemma 3(c) together with standard estimates, we then prove the absolute and locally uniform convergence of the product in (5) from which the assertions given after (5) follow.

To verify (6) first recall that by Definition 2

$$
g_{n}(z, w)=B_{z}(w) \exp h_{n}(z, w)
$$

so

$$
\log \left|g_{n}(z, w)\right|=\log \left|B_{z}(w)\right|+\operatorname{Re} h_{n}(z, w)
$$


Using Lemma 3 together with the remark following Lemma 1 we conclude that

$$
\Delta_{z} \log \left|g_{n}(z, w)\right|=2 \pi\left(\delta_{0}+\delta_{w}\right)-4(n+1) \operatorname{Re} \frac{\left(1-|z|^{2}\right)^{n}}{(1-\bar{z} w)^{n+2}}
$$

(where $\delta_{a}$ denotes the Dirac- $\delta$ measure at $a$ ).

Now Lemma 2 together with (c) yields that for all $w \in U$

$$
\begin{aligned}
& \sum_{k} \log \left|g_{n}\left(z_{k}, w\right)\right|=\frac{1}{2 \pi} \int_{U} \Delta_{z} \log \left|g_{n}(z, w)\right| \log |f(z)| d A(z) \\
& =\log |f(0)|+\log |f(w)|-\frac{2(n+1)}{\pi} \operatorname{Re} \int \frac{\left(1-|z|^{2}\right)^{n}}{(1-\bar{z} w)^{n+2}} \log |f(z)| d A(z) .
\end{aligned}
$$

Putting $w=0$ in this formula we find that

$$
\log |f(0)|=\frac{1}{2} \sum_{k} \log \left|g_{n}\left(z_{k}, 0\right)\right|+\frac{n+1}{\pi} \int \log |f(z)|\left(1-|z|^{2}\right)^{n} d A(z) .
$$

Inserting the last equality in (7) we quickly arrive at the conclusion of the proof, namely (6).

Note that analytic completion of (6) gives the natural factorization

$$
f(w)=e^{i \alpha} \frac{P(w)}{\sqrt{P(0)}} \exp \left\{\int K_{n}(w, z) \log |f(z)|\left(1-|z|^{2}\right)^{n} d(A(z)\}\right.
$$

where $0 \leq \alpha<2 \pi$ and $K_{n}$ is as in (2). This is clearly an analogue of the classical inner-outer factorization, where the inner part of $f$ has been replaced by the Weierstrass-type product $P(w)$.

In order to treat the more general case where we allow $f(0)=0$, and also to recover the result of Andersson ([1], page 147), we proceed as follows. Using the notation of Proposition 1 we note that by Definition 2 and (5)

$$
\sqrt{P(0)}=\prod_{k=1}^{\infty}\left(\left|z_{k}\right| \exp \sum_{\ell=1}^{n+1} \frac{\left(1-\left|z_{k}\right|^{2}\right)^{\ell}}{2 \ell}\right)
$$

Thus

$$
\frac{P(w)}{\sqrt{P(0)}}=\prod_{k=1}^{\infty} \frac{\bar{z}_{k}}{\left|z_{k}\right|} \frac{z_{k}-w}{1-\bar{z}_{k} w} \exp \left(\sum_{\ell=1}^{n+1} \frac{\left(1-\left|z_{k}\right|^{2}\right)^{\ell}}{\ell}\left(\frac{1}{\left(1-\bar{z}_{k} w\right)^{\ell}}-\frac{1}{2}\right)\right) .
$$

Letting $z_{k} \rightarrow 0$ in the general term of the above product we find that the appropriate factor for a zero at the origin is

$$
w \exp \sum_{\ell=1}^{n+1} \frac{1}{2 \ell} .
$$

This function can also be obtained by directly calculating the integral in (6) for the function $f(z)=z$.

Proposition 2. Let $f \in \mathbb{A}_{p, n}$ for some $p>0$ and some integer $n \geq 0$. Assume that $f$ has a zero of order $m \geq 0$ at the origin and that $\left\{z_{k}\right\}$ denotes the remaining 
zeros of $f$ in $U$, repeated according to their multiplicities. Now define

$$
\begin{aligned}
Q(w) & =\left(w^{m} \exp \sum_{\ell=1}^{n+1} \frac{m}{2 \ell}\right) \prod_{k=1}^{\infty} \frac{\bar{z}_{k}}{\left|z_{k}\right|} \frac{z_{k}-w}{1-\bar{z}_{k} w} \\
& \cdot \exp \left(\sum_{\ell=1}^{n+1} \frac{\left(1-\left|z_{k}\right|^{2}\right)^{\ell}}{\ell}\left(\frac{1}{\left(1-\bar{z}_{k} w\right)^{\ell}}-\frac{1}{2}\right)\right) .
\end{aligned}
$$

Then

$$
\frac{f(w)}{Q(w)}=-e^{i \alpha} \exp \left\{\int_{U} K_{n}(w, z) \log |f(z)|\left(1-|z|^{2}\right)^{n} d A(z)\right\}
$$

where

$$
K_{n}(w, z)=\frac{n+1}{\pi}\left(\frac{2}{(1-\bar{z} w)^{n+2}}-1\right),
$$

is the analytic projector for $L^{2}\left(\left(1-|z|^{2}\right)^{n} d A(z)\right)$.

Proof. In light of (9) this follows directly from (8).

Corollary. Let $f \in \mathbb{A}_{p, n}$ for some $p>1$ and some integer $n \geq 0$. Define $Q$ as in the theorem. Then we have a factorization

$$
f(z)=g(z) Q(z)
$$

where $g(z)=\frac{f(z)}{Q(z)}$. Both $g$ and $Q$ belong to $\mathbb{A}_{p, n}$ and for $p=2$

$$
\|f\|_{\mathbb{A}_{2, n}}^{2}=\|g\|_{\mathbb{A}_{2, n}}^{2}+\|Q\|_{\mathbb{A}_{2, n}}^{2} .
$$

Proof. By our theorem, $\log g$ is just the projection of $\log |f|$ by the kernel $K_{n}$. In [3] it was shown that $K_{n}$ induces a bounded operator on $L^{p}\left(\left(1-|z|^{2}\right)^{n} d A(z)\right)$ when $1<p<\infty$. It follows that if $f \in \mathbb{A}_{p, n}(1<p<\infty)$, then $g \in \mathbb{A}_{p, n}$ and consequently $Q \in \mathbb{A}_{p, n}$ also. For $p=2$ we need only remark that just as $\log g$ is the analytic projection of $\log |f|$, so is $\log |g|$ the harmonic projection of $\log |f|$, as seen in (6). Since this is an orthogonal projection, (10) follows from the definition of the norm in $\mathbb{A}_{2, n}$.

\section{REFERENCES}

1. M. Andersson, Solution formulas for the $\partial \bar{\partial}$ equation and weighted Nevanlinna classes in the polydisc, Bull. Soc. Math. France 109 (1985), 135-154. MR 87b:32030

2. M. Andersson, Values in the interior of the $L^{2}$-minimal solutions of the $\partial \bar{\partial}$ equation in the unit ball of $C^{n}$, Publ. Mat. Barcelona 32 (2) (1988), 179-189. MR 90c:32028

3. D. Bekolle, Inégalités à poids pour le projecteur de Bergman dans la boule unité de $C^{n}$, Studia Math. 71 (1981), 305-323. MR 83m:32004

4. E. Beller, Zeros of $A^{p}$ functions and related classes of analytic function, Israel J. Math. 22 (1975), 68-80. MR 52:5973

5. E. Beller, Factorization for non-Nevanlinna classes of analytic functions, Israel J. Math. 27 (1977), 320-330. MR 56:620

6. J. Bruna and J. Ortega-Cerda, On $L^{p}$ solutions of the Laplace equation and zeros of holomorphic functions (to appear).

7. F. Forelli and W. Rudin, Projections on spaces of holomorphic functions in balls, Indiana Univ. J. 24,6 (1974), 593-602. MR 50:10332

8. A. Heilper, The zeros of functions in Nevanlinna's area class, Israel J. Math. 34 (1979), 1-11. MR 82c:30046 
9. C. Horowitz, Zero sets and radial zero sets in function spaces, J. Analyse Math. 65 (1995), 145-159. MR 96h:30066

10. A. L. Shields and D. L. Williams, Bounded projections, duality and multipliers in spaces of analytic functions, Trans. Amer. Math. Soc. 162 (1971), 287-302. MR 58:7053

Department of Mathematics, Bar-Ilan University, Ramat-Gan, Israel

E-mail address: horowitz@macs.biu.ac.il 